\title{
Cayley graphs with an infinite Heesch number
}

\author{
Azer Akhmedov \\ Department of Mathematics \\ North Dakota State University \\ Fargo, ND, U.S.A. \\ azer.akhmedov@ndsu.edu
}

Submitted: Mar 19, 2015; Accepted: Feb 13, 2016; Published: Feb 19, 2016

Mathematics Subject Classifications: 05C25, $05 \mathrm{C} 63$

\begin{abstract}
We construct a 2-generated group $\Gamma$ such that its Cayley graph possesses finite connected subsets with arbitrarily large finite Heesch number. Thus we obtain an example of a Cayley graph with an infinite Heesch number.
\end{abstract}

Keywords: Cayley graphs; Heesch number.

\section{Introduction}

A Heesch number of a polygon $P$ is the maximum number of layers of polygons isometric to $P$ that can surround $P$ without overlapping. For example, a rectangle in the plane has Heesch number infinity since it actually tiles the entire plane. On the other hand, there exist polygons which form only a partial tile for the plane, hence they have a finite Heesch number. It is more interesting to find polygons with a finite Heesch number so we will drop the perfect tiles of the plane from the consideration.

The term Heesch number is named after the geometer Heinrich Heesch who found an example of a polygon with Heesch number 1 (See [5], p.23). This polygon is described in Figure $1^{1}$, and consists of a union of a square, an equilateral triangle and a right triangle with angles 30-60-90. It is already much harder to find polygons with a Heesch number 2 (a well known example of such a polygon is given in Figure 2).

The first examples of polygons with Heesch numbers 2, 3, 4 and 5 have been first discovered by A.Fontaine [3], R.Ammann, F.W.Marshall and C.Mann [6] respectively (See [7] for the history of this problem). DeWeese and Coronaldi have reported (unpublished) the existence of hexagons marked with generalized edge matching rules (i.e. imposing 

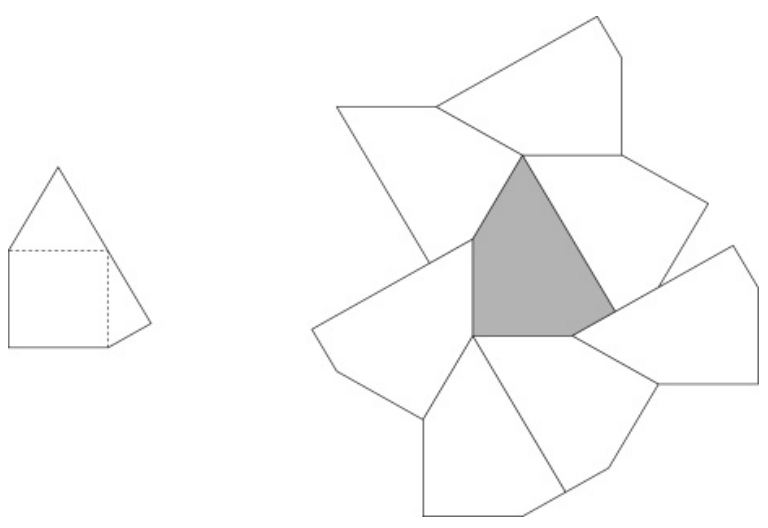

Figure 1: A pentagon with Heesch number 1.

extra conditions on the tiling) having Heesch numbers 0-9 and 11.

Without assuming any condition on the tiling, it is not known if there exists any polygon with a finite Heesch number $N>5$. This is called Heesch's Problem and has close connections to a number of problems in combinatorial geometry such as Domino Problem and Einstein Problem. The latter asks if there exists a tile of the plane consisting of a single polygon $P$ such that any tiling of the plane by $P$ is nonperiodic ("Ein Stein" stands for "one stone" in German; this word play is attributed to Ludwig Danzer). Such polygons have been constructed by G.Margulis and S.Mozes [8] in the hyperbolic plane, while in the Euclidean plane no such examples are known. (The famous Penrose tiling is known to be always nonperiodic, but it uses two polygons, not one. Also, J.Socolar and J.Taylor [9, 10], have found an aperiodic hexagon in the Euclidean plane but again with some extra matching rules.) Incidentally, in the hyperbolic plane $\mathbb{H}^{2}$, the Heesch's Problem is solved completely; it is shown by A.S.Tarasov [11] that there exist polygons in $\mathbb{H}^{2}$ with an arbitrary Heesch number $N \geqslant 1$.

In the Euclidean plane, one can also try to work with its lattice $\mathbb{Z}^{2}$ noticing that any finite connected set $K$ in the Cayley graph of $\mathbb{Z}^{2}$ with respect to the standard generating set $\{( \pm 1,0),(0, \pm 1)\}$ gives rise to the polygon

$$
P(K)=\left\{(x, y) \in \mathbb{R}^{2} \mid \min _{(u, v) \in K} \max \{|x-u|,|y-v|\} \leqslant \frac{1}{2}\right\}
$$

which consists of the $\frac{1}{2}$-neighborhood of the discrete set $K$ in the $l^{\infty}$-metric. Then one can ask if there exist polygons of the form $P(K)$ with a big Heesch number. Indeed, the polygon with Heesch number 2 constructed in [3] is of the form $P(K)$ but this is not the case for the known examples of polygons with Heesch numbers 3, 4 and 5. Notice also that if $K \subset \mathbb{Z}^{2}$ is not connected then $P(K)$ is not a polygon any more, hence restricting to connected sets is natural. We also would like to recall that if one allows disconnected

\footnotetext{
${ }^{1}$ Figure 1 and Figure 2 in this paper have been borrowed from the web page http://math.uttyler.edu/cmann/math/heesch/heesch.htm
} 


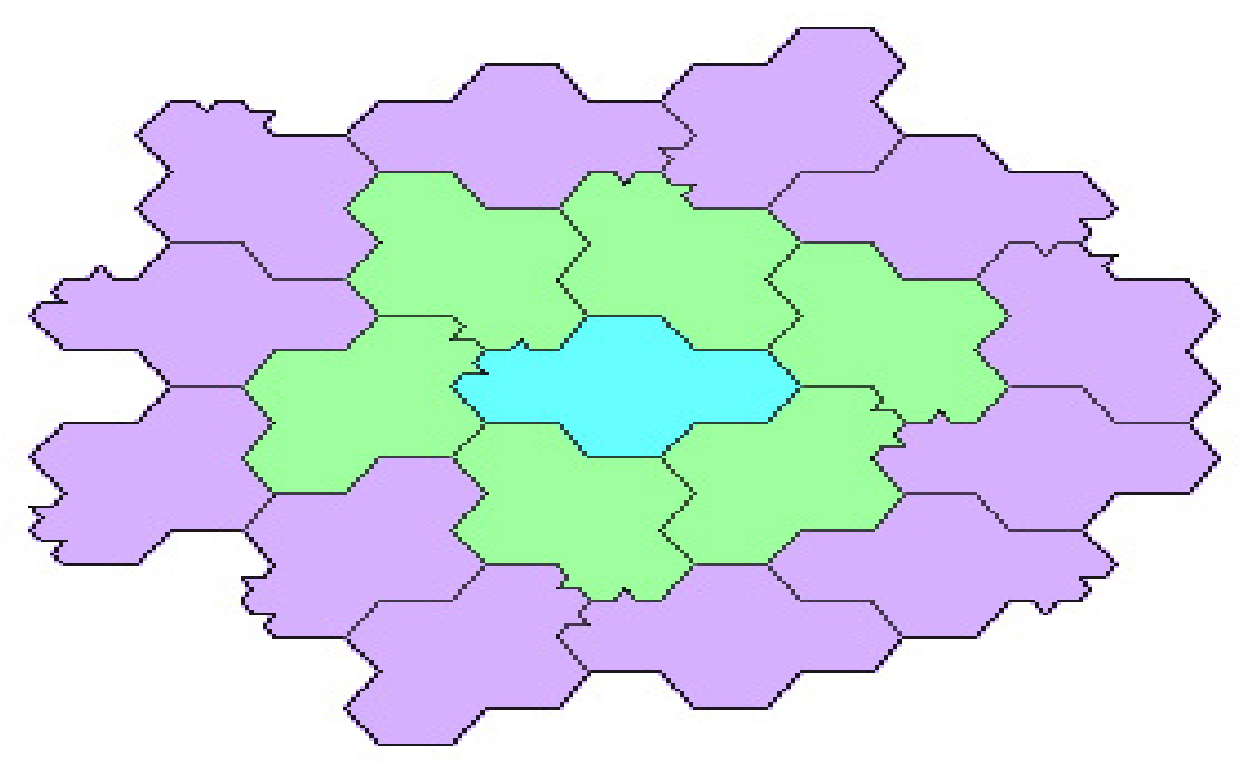

Figure 2: C.Mann's example of a 20-gon with Heesch number 2.

polygons (i.e. union of finitely many connected ordinary polygons) then the Einstein Problem is already solved, see [9].

The notion of a partial tile can be defined in any group (where one moves the sets around by a left translation), and more generally in any graph (where one moves the sets around by an automorphism of the graph). It becomes interesting then if there exists a homogeneous graph (e.g. a vertex transitive graph), and more specifically, a Cayley graph which possesses connected partial tiles with arbitrarily big Heesch number. In the current paper we provide a positive answer to this question; we construct a group $\Gamma$ generated by a two element subset $S$ such that the Cayley graph of $\Gamma$ with respect to $S$ possesses partial tiles of arbitrarily big finite Heesch number (in the Cayley graph, the sets are moved around by left translations of the group). Notice that it is very easy to find disconnected sets with a big finite Heesch number, so without the connectedness condition the question is easy (and somewhat unnatural).

To state our main result, we need to define the notions of tile, partial tile, and Heesch number in the setting of an arbitrary finitely generated group.

Definition 1. (tiles) Let $\Gamma$ be a countable group, $F$ be a finite subset with cardinality at least 2. $F$ is called a tile if there exists $C \subset \Gamma$ such that $\Gamma=\sqcup_{g \in C} g F$. The partition $\sqcup_{g \in C} g F$ is called a tiling, and the set $C$ is called the center set of this tiling. We will always assume that $1 \in C$.

We will also be interested in partial tiles of groups.

Definition 2. (partial tiles) Let $\Gamma$ be a countable group, $F$ be a finite subset with cardinality at least 2 such that $F$ does not tile $\Gamma$. For a subset $M$ of $\Gamma$, we say $F$ 
tiles $M$ if there exists $C \subset \Gamma$ such that $1 \in C$ and $M \subseteq \sqcup_{g \in C} g F$. F will be called a partial tile of $\Gamma$, and the partition $\underset{g \in C}{\sqcup} g F$ is called a partial tiling.

Let now $\Gamma$ be a finitely generated group. We will fix a finite symmetric generating set $S$ of $\Gamma$, and study partial tiles in the Cayley graph of $\Gamma$ w.r.t. the left invariant Cayley metric given by $S$ (by a set in a Cayley graph we mean a set of vertices). We will always assume that the generating set does not include the identity element so, in particular, the Cayley graphs do not have loops. For all $g \in \Gamma,|g|_{\Gamma}$ will denote the length of the element $g$ in the Cayley metric, and for all $x, y \in G, d_{\Gamma}(x, y)$ will denote the distance between $x$ and $y$, i.e. $d_{\Gamma}(x, y)=\left|x^{-1} y\right|$ (we will drop the index if it is clear from the context which group we are considering). For any $g \in \Gamma$, we will also write $B_{g}(r)=\{x \in \Gamma \mid d(g, x) \leqslant r\}$ for the ball of radius $r$ around $g$; for any two subsets $A, B$ of $\Gamma$ we will write $d(A, B)=\min _{x \in A, y \in B} d(x, y)$ for the distance between the sets $A$ and $B$; and for any finite subset $A \subset \Gamma$, we will also write $\partial A=\left\{x \in A|| B_{1}(x) \cap(\Gamma \backslash A)|=| B_{1}(x) \mid-2\right\}$ for the boundary of $A$; so the boundary of $A$ consists of those points $x$ of $A$ such that exactly one neighbor of $x$ belongs to $A$. Finally, a set $F$ in a Cayley graph will be called connected if for any $a, b \in F$, there exists a subset $\left\{z_{1}, \ldots, z_{n}\right\} \subseteq F$ such that $z_{1}=a, z_{n}=b$ and $d\left(z_{i}, z_{i+1}\right)=1,1 \leqslant i \leqslant n-1$.

If $\alpha=\left(x_{1}, \ldots, x_{n}\right), \beta=\left(y_{1}, \ldots, y_{m}\right)$ are paths in a Cayley graph then we say $\beta$ is quasitransversal to $\alpha$ if there exists $p \in\{2, \ldots, n-1\}$ such that $y_{1}=x_{n}, y_{2}=x_{n-1}, \ldots, y_{n-p+1}=$ $x_{p}$ and $y_{i} \notin\left\{x_{1}, \ldots, x_{n}\right\}$ for all $i \geqslant n-p$. If $\beta$ is quasi-transversal to $\alpha$ as above, then the path $\left(y_{1}, \ldots, y_{n-p}, y_{n-p+1}\right)$ will be called the head of $\beta$ (w.r.t. $\alpha$ ), and the path $\left(y_{n-p+1}, \ldots, y_{m}\right)$ will be called the tail of $\beta$ (w.r.t. $\left.\alpha\right)$. We also will say that the divergence of $\alpha$ and $\beta$ occurs at $x_{p}$. We will say $\beta$ contains $\alpha$ if $\left\{x_{1}, \ldots, x_{n}\right\} \subseteq\left\{y_{1}, \ldots, y_{m}\right\}$; and $\beta$ follows $\alpha$ if $y_{1}=x_{n}$ and $\left\{x_{1}, \ldots, x_{n-1}\right\} \cap\left\{y_{1}, \ldots, y_{n}\right\}=\emptyset$. The length of $\alpha$ will be defined as $|\alpha|=\left|x_{1}^{-1} x_{n}\right|$.

For a partial tile $K$, we say that the Heesch number of $K$ equals $N$, if one can tile $N$ layers around $K$ but not $N+1$ layers. To be precise, we need the following definitions.

Definition 3. (layers) Let $C \subseteq \Gamma$ such that $\pi=\sqcup_{g \in C} g K$ is a partial tiling. Let also $C_{0} \subseteq C$. We say $C_{0} K$ is the layer of level 0 of $\pi$ if $C_{0}=\{1\}$. For a subset $C_{1} \subseteq C$, we say $C_{1} K$ is the layer of level 1 of $\pi$, if $C_{1}$ is a minimal subset of $C$ such that $C_{1} \cap C_{0}=\emptyset$ and $\{x \in \Gamma \mid d(x, K)=1\} \subseteq C_{1} K$. (notice that if $C_{1}$ exists then it is unique). For any $n \geqslant 2$, inductively, we define the layer of level $n$ of $\pi$ as follows: if $C_{0} K, C_{1} K, \ldots, C_{n-1} K$ are the layers of level $0,1, \ldots, n-1$ respectively, then we say $C_{n} K$ is the layer of level $n$ if $C_{n}$ is a minimal subset of $C$ such that

$$
C_{n} \cap \underset{0 \leqslant i \leqslant n-1}{\sqcup} C_{i}=\emptyset \text { and }\left\{x \in \Gamma \mid d\left(x, \underset{0 \leqslant i \leqslant n-1}{\sqcup} C_{i} K\right)=1\right\} \subseteq C_{n} K
$$

Motivated by the above definitions, one naturally defines the notion of a Heesch number for partial tiles of the group $\Gamma$.

Definition 4. (Heesch number) Let $K$ be a finite subset of $\Gamma$. We write $\operatorname{Heesch}(K)=N$ if $N$ is the maximal non-negative integer such that $\Gamma$ has a partial tiling by the left shifts of $K$ which has $N$ layers. 
If a finite subset $K$ tiles $\Gamma$ then we will write $\operatorname{Heesch}(K)=\infty$.

The main result of the paper is the following

Theorem 5. There exists a finitely generated group $\Gamma$ with a fixed finite generating set $S$ such that for any natural $N, \Gamma$ has a connected partial tile $K_{N}$ with a finite Heesch number bigger than $N$.

Let us emphasize again that without the condition "connected" the result would be trivial as the group $\mathbb{Z}$ easily possesses disconnected partial tiles with arbitrarily big finite Heesch number. The statement of Theorem 5 also motivates the notion of a Heesch number for an arbitrary Cayley graph $\mathcal{G}=\mathcal{G}(\Gamma, S)$ of a group $\Gamma$ with respect to the symmetric generating set $S$, namely, we define this number as $\operatorname{Heesch}(\mathcal{G}(\Gamma, S))=\sup \mathcal{H}(\Gamma, S)$ where

$\mathcal{H}(\Gamma, S)=\left\{N \geqslant 0 \mid \mathcal{G}(\Gamma, S)\right.$ admits a connected partial tile $K_{N}$ with $\left.\operatorname{Heesch}\left(K_{N}\right)=N\right\}$.

Thus, if $\mathcal{G}(\Gamma, S)$ possesses connected partial tiles with arbitrarily large Heesch number $N$, then we will have Heesch $(\mathcal{G}(\Gamma, S))=\infty$. (Also, if all finite connected subsets of $\Gamma$ actually tile $\Gamma$ then $\mathcal{H}(\Gamma, S)=\emptyset$, and we will have $\operatorname{Heesch}(\mathcal{G}(\Gamma, S))=-\infty$; here, we follow a convention that $\sup \emptyset=-\infty)$. Notice that the quantity $\operatorname{Heesch}(\mathcal{G}(\Gamma, S))$ is quite sensitive to the choice of the generating set $S$.

Theorem 5 now implies the existence of a finitely generated group $\Gamma$ such that its Cayley graph $\mathcal{G}(\Gamma, S)$ with respect to a certain finite symmetric generating set $S$ has an infinite Heesch number, i.e. $\operatorname{Heesch}(\mathcal{G}(\Gamma, S))=\infty$.

Remark 6. Let $\mathcal{G}(\Gamma, S)$ be the Cayley graph of $\Gamma$ with respect to a finite symmetric generating set $S$. One can consider the automorphism group $\operatorname{Aut}(\mathcal{G}(\Gamma, S))$ of the Cayley graph. Notice that this automorphism group contains an isomorphic copy of the group $\Gamma$ since the latter acts on the Cayley graph by left translations. Then for any finite connected set $F$ of the Cayley graph $\mathcal{G}(\Gamma, S)$, one can introduce the notion of partial tiles and Heesch numbers similarly; for $g \in A u t(\mathcal{G}(\Gamma, S))$ and $x \in \Gamma$, the expression $g x$ will be understood as the image of the automorphism $g$ applied to $x$ (instead of the left translation of $x$ by $g$ ). The main result of the paper, i.e. Theorem 5 holds in this general setting as well by the same proof adapted to this new language.

Example 7. Considering the full automorphism groups of Cayley graphs (instead of just the left translations) allows more possibilities for constructing polyhedral shapes of finite Heesch number in the spaces where the Cayley graphs embed. Following A.Fontaine, we consider a subset

$$
K=\{(i, 0) \mid 0 \leqslant i \leqslant 10\} \sqcup\{(i, 1) \mid 0 \leqslant i \leqslant 3\} \sqcup\{(i, j) \mid 7 \leqslant i \leqslant 10,1 \leqslant j \leqslant 2\}
$$

of $\mathbb{Z}^{2}$ viewed also as a subset of the Cayley graph $\mathcal{G}$ of $\mathbb{Z}^{2}=\langle a, b\rangle$ with respect to the standard generating set $\left\{a^{ \pm 1}, b^{ \pm 1}\right\}$. The automorphisms of $\mathcal{G}$ include all translations by the group elements as well as some reflections and rotations (such as reflections w.r.t. coordinate axis or rotations by multiples of 90 degrees). Let $R_{x}, R_{y}$ denote the reflections 
w.r.t. $x$-axis and $y$-axis respectively, and let $r$ be a rotation by 90 degrees in the clockwise direction. We consider the following sets of automorphisms of $\mathcal{G}$ :

$$
\begin{gathered}
C_{0}=\{1\}, \\
C_{1}=\left\{a^{7} b^{12} R_{y} r, a^{7} b^{14} r, a^{12} b^{7} R_{y} r, a^{9} r^{2}, a^{-3} b^{-7} R_{y} r^{-1}, a^{-7} b^{5} R_{x}\right\}, \\
C_{2}=\left\{a^{-5} b^{5}, a^{-6} b^{8}, a^{-6} b^{11}, a^{7} b^{12} r^{-1}, a^{7} b^{14} R_{y} r^{-1}, a^{20} b^{13} R_{y}, a^{20} b^{10} R_{y},\right. \\
a^{19} b^{10} r^{2}, a^{12} b^{8} r, a^{16} b^{-5} R_{y}, a^{2} b^{-12} R_{y} r^{-1}, a^{-1} b^{-14} R_{y} r^{-1}, a^{-1} b^{-7} R_{y} r, \\
\left.a^{-3} b^{-8} r^{-1}, a^{-6} b^{-8} r^{-1}, a^{-7} b^{5} r^{2}, a^{-5} b^{5} R_{y}\right\} .
\end{gathered}
$$

Then $C_{1} K$ forms a layer around $C_{0} K=K$ and $C_{2} K$ forms a layer around $C_{1} K$. It is impossible to make a partial tiling with three layers. Thus by the extended definition in Remark $6, K$ has a Heesch number two. The three sets $\underset{g \in C_{i}}{\cup} P(g K), 0 \leqslant i \leqslant 2$ also form a partial tiling of the polygon $P(K)$ in the Euclidean plane.

\section{Hyperbolic Limits}

We will be using the well known concept of hyperbolic limits. The reader may consult with [1] for basic notions of the theory of word hyperbolic groups but we will assume nothing other than the familiarity with the definition of a word hyperbolic group. Following the convention, we will say that a word hyperbolic group is elementary if it is virtually cyclic. Let us first recall a well known theorem due to Gromov and Delzant which motivates the notion of hyperbolic limit.

Theorem 8. (See [4] and [2]) Let $H$ be a non-elementary word hyperbolic group with a fixed finite generating set. Then for any non-torsion element $\gamma \in H$ and for any $R>0$ there exists a positive integer $N_{0}$ such that for all $N>N_{0}$ the quotient $H^{\prime}=H /\left\langle\gamma^{N}=1\right\rangle$ is non-elementary word hyperbolic, moreover the quotient map $\pi: H \rightarrow H^{\prime}$ is injective on the ball $B_{R}(1)$ of radius $R$ around the identity element. [in other words, adding the relation $\gamma^{N}=1$ is injective on the ball of radius $R$ and the quotient remains non-elementary word hyperbolic].

Let now $H$ be a non-elementary word hyperbolic group with a fixed finite symmetric generating set $S$. The group $H_{\infty}$ is called a hyperbolic limit of $H$ if there exists a sequence $H_{0}, H_{1}, H_{2}, \ldots$ of non-elementary word hyperbolic groups such that

(i) $H_{0}=H$;

(ii) $H_{n+1}$ is a quotient of $H_{n}$ for all $n \in \mathbb{N} \cup\{0\}$;

(iii) for all $n \in \mathbb{N} \cup\{0\}$, the quotient epimorphism $\pi_{n}: H_{n} \rightarrow H_{n+1}$ is injective on the ball of radius $n+1$ around identity element w.r.t. the generating set $S$ [more precisely, with respect to the generating set $\pi_{n-1} \ldots \pi_{1} \pi_{0}(S)$, but by abusing the notation, we will denote it by $S]$. 
Since the ball of radius $n$ remains injective (unchanged) by all the epimorphisms $\pi_{i}$, $i>n$, the union of these stable balls determines a group, denoted by $H_{\infty}$, and called a hyperbolic limit of $H$. If $g \in H_{n}$ belongs to the ball of radius $n$ around the identity element then, by abusing the notation, we will denote the image of $g$ in $H_{\infty}$ by $g$. So the image of the generating set $S$ of $H$ in $H_{\infty}$ will be denoted by $S$.

A non-elementary word hyperbolic group may have many different hyperbolic limits, and groups which are very far from being word hyperbolic can be hyperbolic limits. Hyperbolic limits are very useful; for example, using Theorem 8, one immediately obtains a finitely generated infinite torsion group as a hyperbolic limit of an arbitrary non-elementary word hyperbolic group.

In the proof of Theorem 5, the group $\Gamma$ will be constructed as a hyperbolic limit of virtually free groups. Having a virtually free group at each step allows a great simplification in the argument but that also means we need to make extra efforts to keep the group virtually free at each step. Indeed, with a much more complicated argument, starting with an arbitrary non-elementary word hyperbolic group $H_{0}$, one can construct a group as a quotient of $H_{0}$ with connected partial tiles of arbitrarily big finite Heesch number.

\section{Intermediate Results}

The following simple lemma will be extremely useful.

Lemma 9. Let $F$ be a finite subset of $\Gamma$, and $\pi: \Gamma \rightarrow \Gamma^{\prime}$ be an epimorphism such that $\pi(F)$ is a tile of $\Gamma^{\prime}$ and $|F|=|\pi(F)|$. Then $F$ is a tile of $\Gamma$.

Proof. Assume $N=\operatorname{ker}(\pi)$, and $\Gamma^{\prime}=\underset{g^{\prime} \in C^{\prime} \subseteq \Gamma^{\prime}}{\sqcup} g^{\prime} \pi(F)$ is a partition of $\Gamma^{\prime}$ into tiles. For every $g^{\prime} \in C^{\prime}$ we choose a representative $g \in \Gamma$ with $\pi(g)=g^{\prime}$. Let $C$ be the set of all representatives. Then we have a partition $\Gamma=\underset{g \in C, n \in N}{\sqcup} g n F$.

In the proof of the main theorem, we will be considering virtually free groups. If $G$ is such a group with a fixed Cayley metric |.|, and $N$ is finite index free subgroup of rank $r \geqslant 2$ with a generating set $S$ of cardinality $r$, then, in general, it is possible that $|g|<|s|$ where $s \in S$ while $g \in N \backslash\left(\{1\} \cup S \cup S^{-1}\right)$. However, we can avoid this situation by taking $N$ to be a very deep (still of finite index) subgroup in $G$. The following lemma will be useful.

Lemma 10. Let $G$ be a virtually non-abelian free group with a fixed finite generating set and the corresponding left-invariant Cayley metric |.|. Then there exists a finite index normal subgroup $N \unlhd G$ such that the following conditions hold:

(c1) $N$ is a free group of rank $k \geqslant 2$ generated by a subset $\left\{g_{1}, \ldots, g_{k}\right\}$,

(c2) $\min \left\{\left|g_{1}\right|, \ldots,\left|g_{k}\right|\right\} \leqslant 1.1 \min \{|x|: x \in N \backslash\{1\}\}$.

Proof. Since $G$ is virtually free, with respect to the metric |.|, it is $\delta$-hyperbolic for some $\delta>0$ (i.e. geodesic triangles are $\delta$-thin, see [1]). Let $G_{0}$ be a free subgroup of $G$ of finite index such that $G_{0} \cap B_{R_{0}}(1)=\{1\}$ where $R_{0}>100 \delta$. Let also $r=\operatorname{rank}\left(G_{0}\right)$ and 
$S_{0}=\left\{f_{1}, \ldots, f_{r}\right\}$ be a generating set of $G_{0}$. We will denote the left invariant Cayley metric of $G_{0}$ with respect to $S_{0}$ by |.|. . (It is well known that the metrics |.| and |.| $\left.\right|_{0}$ are bi-Lipshitz equivalent, i.e. there exists $\lambda>1$ such that for all $x \in G_{0}, \frac{1}{\lambda}|x|_{0} \leqslant|x| \leqslant \lambda|x|_{0}$. However, we will need a finer analysis to get stronger inequalities.)

$G_{0}$ has a finite index normal subgroup $N$ such that $N$ is a normal subgroup of $G$. Let $N \cap B_{R}(1)=\{1\}$ for some $R>R_{0}$. Let also $k=\operatorname{rank} N$ and

$$
\mathcal{A}=\left\{\left(\gamma_{1}, \ldots, \gamma_{k}\right):\left\langle\gamma_{1}, \ldots, \gamma_{k}\right\rangle=N,\left|\gamma_{1}\right|_{0} \leqslant\left|\gamma_{2}\right|_{0} \leqslant \ldots \leqslant\left|\gamma_{k}\right|_{0}\right\} .
$$

Now we choose a suitable generating $k$-tuple of $N$, i.e. an arbitrary $\left(g_{1}, \ldots, g_{k}\right) \in \mathcal{A}$ such that for any $\left(\gamma_{1}, \ldots, \gamma_{k}\right) \in \mathcal{A}$ we have $\left|g_{1}\right|_{0} \leqslant\left|\gamma_{1}\right|_{0}$, moreover, for all $i \in\{1, \ldots, k-1\}$, if $\left(g_{1}, \ldots, g_{i}, \gamma_{i+1}, \ldots, \gamma_{k}\right) \in \mathcal{A}$ then $\left|g_{i+1}\right|_{0} \leqslant\left|\gamma_{i+1}\right|_{0}$.

We will first prove the claim (a somewhat stronger version of it) for the group $G_{0}$ in the |. $\left.\right|_{0}$ metric, more precisely, we will show that for all $g \in N \backslash\{1\}$ such that $|g|_{0}=\min \left\{|x|_{0}\right.$ : $x \in N \backslash\{1\}\}$ we have $|g|_{0}=\left|g_{1}\right|_{0}$. We can write $g$ as a reduced word $W\left(g_{1}, \ldots, g_{k}\right)$ in the alphabet $\left\{g_{1}^{ \pm 1}, \ldots, g_{k}^{ \pm 1}\right\}$ such that the length of $g$ in this alphabet is minimal; we will denote this length by $q$. Each of $g_{i}^{ \pm 1}, 1 \leqslant i \leqslant k$ and the element $g$ itself can be represented as reduced word in the alphabet $\left\{f_{1}^{ \pm 1}, \ldots, f_{r}^{ \pm 1}\right\}$ as well. In the Cayley graph of $G$ w.r.t. this generating set, $W$ can be represented as a concatenation $\gamma_{1} \gamma_{2} \ldots \gamma_{q}$ of the paths $\gamma_{i}, 1 \leqslant i \leqslant q$, where the path $\gamma_{i}$ represents the $i$-th letter of the word $W\left(g_{1}, \ldots, g_{k}\right)$ (in the alphabet $\left\{g_{1}^{ \pm 1}, \ldots, g_{k}^{ \pm 1}\right\}$ ).

By the choice of the generating $k$-tuple $\left(g_{1}, \ldots, g_{k}\right)$ and by the minimality assumption on $|g|_{0}, \gamma_{i+1}$ does not contain $\gamma_{i}, 1 \leqslant i \leqslant q-1$. Then, either $\gamma_{i+1}$ follows $\gamma_{i}$, or $\gamma_{i+1}$ is quasitransversal to $\gamma_{i}$, for all $1 \leqslant i \leqslant q-1$. In the latter case, again by the choice of $\left(g_{1}, \ldots, g_{k}\right)$, we have $\left|\operatorname{tail}\left(\gamma_{i+1}\right)\right|_{0}>\left|\operatorname{head}\left(\gamma_{i+1}\right)\right|_{0}$ (although not really needed, by the minimality of $q$ we can even claim a strict inequality). Moreover, if $\gamma_{i+2}$ is also quasi-transversal to $\gamma_{i+1}$ then the divergence occurs at the tail of $\gamma_{i+1}$, i.e. $\left|\operatorname{head}\left(\gamma_{i+2}\right)\right|_{0}<\left|\operatorname{tail}\left(\gamma_{i+1}\right)\right|_{0}$. From these inequalities we immediately obtain that $|g|_{0} \geqslant\left|g_{1}\right|_{0}$.

Now we need to prove the same inequality (with an extra factor of 1.1.) in the original |.| metric. For this, first, we need to arrange that the generating set $S_{0}=\left\{f_{1}, \ldots, f_{r}\right\}$ we start with is also suitable: let

$$
\mathcal{B}=\left\{\left(\gamma_{1}, \ldots, \gamma_{r}\right):\left\langle\gamma_{1}, \ldots, \gamma_{r}\right\rangle=G_{0},\left|\gamma_{1}\right| \leqslant\left|\gamma_{2}\right| \leqslant \ldots \leqslant\left|\gamma_{k}\right|\right\}
$$

and choose an arbitrary $r$-tuple $\left(f_{1}, \ldots, f_{r}\right) \in \mathcal{B}$ such that for any $\left(\gamma_{1}, \ldots, \gamma_{r}\right) \in \mathcal{B}$ we have $\left|f_{1}\right| \leqslant\left|\gamma_{1}\right|$, moreover, for all $i \in\{1, \ldots, r-1\}$, if $\left(f_{1}, \ldots, f_{i}, \gamma_{i+1}, \ldots, \gamma_{r}\right) \in \mathcal{B}$ then $\left|f_{i+1}\right| \leqslant\left|\gamma_{i+1}\right|$.

Now we choose a suitable generating $k$-tuple of $N$ in the $|$.$| metric, i.e. we will look$ at the following set of $k$-tuples

$$
\mathcal{C}=\left\{\left(\gamma_{1}, \ldots, \gamma_{k}\right):\left\langle\gamma_{1}, \ldots, \gamma_{k}\right\rangle=N,\left|\gamma_{1}\right| \leqslant\left|\gamma_{2}\right| \leqslant \ldots \leqslant\left|\gamma_{k}\right|\right\}
$$

and choose an arbitrary $k$-tuple $\left(g_{1}, \ldots, g_{k}\right) \in \mathcal{C}$ such that for any $\left(\gamma_{1}, \ldots, \gamma_{k}\right) \in \mathcal{C}$ we have $\left|g_{1}\right| \leqslant\left|\gamma_{1}\right|$, moreover, for all $i \in\{1, \ldots, k-1\}$, if $\left(g_{1}, \ldots, g_{i}, \gamma_{i+1}, \ldots, \gamma_{k}\right) \in \mathcal{C}$ then $\left|g_{i+1}\right| \leqslant\left|\gamma_{i+1}\right|$. 
We will assume that $g \in N \backslash\{1\}$ with $|g|=\min \{|x|: x \in N \backslash\{1\}\}$ and we need to show that $|g| \leqslant 1.1\left|g_{1}\right|$. Again, $g$ can be written as a reduced word $W\left(g_{1}, \ldots, g_{k}\right)=\gamma_{1} \ldots \gamma_{q}$ in the alphabet $\left\{g_{1}^{ \pm 1}, \ldots, g_{k}^{ \pm 1}\right\}$ such that the length $q$ of $g$ in this alphabet is minimal, and $g$ also can be represented as reduced word in the alphabet $\left\{f_{1}^{ \pm 1}, \ldots, f_{r}^{ \pm 1}\right\}$ as well. In this case, we will have the inequalities $\left|\operatorname{tail}\left(\gamma_{i+1}\right)\right|>\left|\operatorname{head}\left(\gamma_{i+1}\right)\right|-10 \delta$ (when $\gamma_{i+1}$ is quasi-transversal to $\gamma_{i}$ ) and $\left|\operatorname{head}\left(\gamma_{i+2}\right)\right|<\left|\operatorname{tail}\left(\gamma_{i+1}\right)\right|+10 \delta$ (when, in addition, $\gamma_{i+2}$ is also quasi-transversal to $\gamma_{i+1}$ ) instead. However, if $R_{0}$ is sufficiently big w.r.t. $\delta$, by the choice of $S_{0}$ and by $\delta$-hyperbolicity, we obtain that, in the Cayley graph of $G_{0}$ w.r.t. $S_{0}$, the path $\gamma_{i+2}$ will not include any neighbor of $x$ in the concatenation $\gamma_{i} \gamma_{i+1}$ where $x$ is an element at which the divergence of $\gamma_{i}$ and $\gamma_{i+1}$ occurs. Thus we again have the inequality $|g| \geqslant\left|g_{1}\right|-20 \delta q$. Then, for sufficiently big $R$ we obtain that $|g| \geqslant \frac{1}{1.1}\left|g_{1}\right|$.

We now would like to state the central result of this section.

Proposition 11. Let $G$ be a finitely generated virtually non-abelian free group, $N$ be a finite index normal subgroup of $G$ such that $N$ is a free group of rank $k \geqslant 4$ generated by elements $g_{1}, \ldots, g_{k}$. Then there exists a generating $k$-tuple $\left(h_{1}, \ldots, h_{k}\right)$ of $N$ such that for all positive real numbers $R>0$, there exists $n_{0} \geqslant 1$ such that for all $n \geqslant n_{0}$, the quotient $G /\left\langle h_{i}^{n}=1,1 \leqslant i \leqslant k\right\rangle$ is a virtually non-abelian free group and the quotient epimorphism $G \rightarrow G /\left\langle h_{i}^{n}=1,1 \leqslant i \leqslant k\right\rangle$ is injective on the ball of radius $R$ around the identity element.

Before proving Proposition 11, we need to make a small digression into the outer automorphisms of free groups explaining also why do we need the condition $k \geqslant 4$. Let $G, N$ be as in Proposition 11, i.e. $G$ is a virtually non-abelian free group, and $N$ is a finite index free normal subgroup. If $g \in N$ then we have $\mathcal{N}_{N}(g) \leqslant \mathcal{N}_{G}(g) \leqslant N$ but the normal closure $\mathcal{N}_{G}(g)$ of $g$ in $G$ can be much bigger than the normal closure $\mathcal{N}_{N}(g)$ of $g$ in $N$. This is an undesirable situation for us; however, one can replace $\left(g_{1}, \ldots, g_{k}\right)$ with another generating $k$-tuple $\left(h_{1}, \ldots, h_{k}\right)$ of $N$, and let $g$ be quite special by taking $g=h_{1}^{m}$, for some (sufficiently big) $m \geqslant 1$. Then we can take the normal closure of a more symmetric set $S=\left\{h_{1}^{m}, \ldots, h_{k}^{m}\right\}$, and it turns out that if $k \geqslant 4$ then the normal closure of $S$ in $N$ coincides with its normal closure in $G$. To see this let us first recall the following nice result of B. Zimmerman [12].

Theorem 12. Let $k \geqslant 2$. A finite subgroup Out $\left(\mathbb{F}_{k}\right)$ has a maximal order 12 for $k=2$, and a maximal order $2^{k} k$ ! for $k \geqslant 3$. Moreover, for $k \geqslant 4$, all finite subgroups are conjugate to a unique maximal subgroup $H_{k}$ of order $2^{k} k$ !.

If $a_{1}, \ldots, a_{k}$ are some generators of $\mathbb{F}_{k}$ then let

$$
L_{k}=\left\{\phi \in \operatorname{Aut}\left(\mathbb{F}_{k}\right) \mid \forall i, \phi\left(a_{i}\right) \in\left\{a_{1}^{ \pm 1}, \ldots, a_{k}^{ \pm 1}\right\} .\right.
$$

Notice that $L_{k}$ is a subgroup of $A u t\left(\mathbb{F}_{k}\right)$ of order exactly $2^{k} k$ !. Moreover, the group $L_{k}$ induces a finite subgroup $\overline{L_{k}}$ of $\operatorname{Out}\left(\mathbb{F}_{k}\right)$ of the same cardinality. Then by Theorem 12 , for $k \geqslant 4$, all finite subgroups of $\operatorname{Out}\left(\mathbb{F}_{k}\right)$ are conjugate to a subgroup of $\overline{L_{k}}$ (in other words, in the statement of Theorem 12, one can take $H_{k}$ to be $\overline{L_{k}}$ ).

Thus we obtain the following lemma. 
Lemma 13. $G$ be a finitely generated virtually non-abelian free group, $N$ be a finite index normal subgroup of $G$ such that $N$ is free of rank $k \geqslant 4$. Then there exists a generating $k$-tuple $\left(h_{1}, \ldots, h_{k}\right)$ of $N$ such that for all $m \geqslant 2$, we have $\mathcal{N}_{N}(S)=\mathcal{N}_{G}(S)$ where $S=\left\{h_{1}^{m}, \ldots, h_{k}^{m}\right\}$.

The Lemma 13 guarantees that $N / \mathcal{N}_{N}(S)$ is a finite index subgroup of $G / \mathcal{N}_{G}(S)$; it remains to recall a folklore result that $N / \mathcal{N}_{N}(S)$ is always virtually free. For the sake of completeness we would like to formalize this claim in the following lemma.

Lemma 14. Let $N \cong \mathbb{F}_{k}$ be a free group of rank $k \geqslant 3$ generated by elements $a_{1}, \ldots, a_{k}$. Then for all $m \geqslant 2$, the quotient group $N_{m}=N /\left\langle a_{1}^{m}=\cdots=a_{k}^{m}=1\right\rangle$ is virtually non-abelian free.

For the proof, it suffices to recall a well known fact that the free product of two finitely generated virtually free groups is still virtually free (in particular, the free product of finite groups is virtually free), moreover, the free product of two non-trivial finite groups is virtually non-abelian free provided at least one of these finite groups have order at least 3 .

Now, Proposition 11 follows immediately from Theorem 8, Lemma 13 and Lemma 14, by taking $m$ to be a sufficiently big positive integer.

We close this section with the following very useful result.

Lemma 15. Let $G$ be a group with a fixed finite symmetric generating set $S$ of cardinality at least four such that there is no non-trivial relation of length less than four among the elements of $S$, and for some $s \geqslant 1$, and for all distinct $x, y, z \in S$ there exists a path in $G \backslash\{1\}$ of length at most $s$ from $x$ to $y$ not containing $z$. Let also $N$ be a finite index normal subgroup of $G$ such that $N \cap B_{10 s}(1)=\{1\}$, and $g \in N \backslash\{1\}$ such that $|g| \leqslant 1.1 \min \{|x| \mid x \in N \backslash\{1\}\}$. Then there exists a connected subset $A \subset G$ such that the following conditions hold:

(a1) $|A|=|G / N|$,

(a2) $1 \in A$,

(a3) $x^{-1} y \notin N$ for all distinct $x, y \in A$,

(a4) $d(g, A)=1$,

(a5) if $\pi=\underset{h \in C}{\sqcup} h A$ is the left tiling of $G$ by $A$ then $g^{n} \in C$ for all $n \in \mathbb{Z} .{ }^{2}$.

Proof. Let $\epsilon: G \rightarrow G / N$ be the quotient map, and $\mathcal{G}, \mathcal{G}_{1}$ be the Cayley graphs of the groups $G, G / N$ with respect to the generating sets $S, \epsilon(S)$ respectively. Let also $r=\left(x_{0}=\right.$ $1, x_{1}, \ldots, x_{n}, x_{n+1}=g$ ) be a path in $\mathcal{G}$ connecting 1 to $g$ such that $\left|x_{i}\right|=i, 0 \leqslant i \leqslant n+1$.

We will first demonstrate the construction of the set $A$ satisfying conditions (a1)-(a4). In the Cayley graph $\mathcal{G}_{1}$, we consider the path $r_{1}=\left(1, \epsilon\left(x_{1}\right), \ldots, \epsilon\left(x_{n}\right)\right)$, and build the subsets $Q_{1}, Q_{2}, \ldots, Q_{|G / N|-n}$ in $G / N$ inductively as follows.

We let $Q_{1}=\left\{1, \epsilon\left(x_{1}\right), \ldots, \epsilon\left(x_{n}\right)\right\}$, and if the sets $Q_{1}, \ldots, Q_{k}$ are already defined for some $k<|G / N|-n$, then we let $Q_{k+1}=Q_{k} \sqcup\left\{z_{k}\right\}$ where $\left|z_{k}^{-1} z\right|=1$ for some $z \in Q_{k}$ (i.e.

\footnotetext{
${ }^{2}$ let us recall that, by definition, the identity element always belongs to the set of centers of the tiling
} 


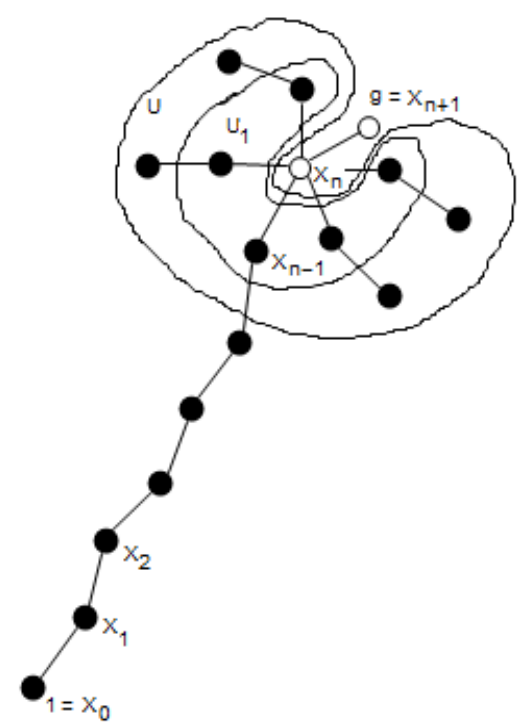

Figure 3: The sets $V_{0}$ and $U$; the elements of $V_{0}$ are represented with black dots

the distance from $z_{k}$ to $Q_{k}$ equals 1 , in the Cayley graph $\left.\mathcal{G}_{1}\right)$. Then $Q_{|G / N|-n}=G / N$, and we start defining $A_{1}, \ldots, A_{|G / N|-n}$ inductively as follows: we let $A_{1}=\left\{1, x_{1}, \ldots, x_{n}\right\}$, and if $A_{1}, \ldots, A_{k}$ are defined for some $k<|G / N|-n$, then we let $A_{k+1}$ to be any connected set in $\mathcal{G}$ such that $A_{k+1}=A_{k} \cup\left\{y_{k}\right\}$ where $y_{k} \in \epsilon^{-1}\left(z_{k}\right)$.

Then the set $A_{|G / N|-n}$ already satisfies conditions (a1)-(a4) of the lemma. To make it satisfy the condition (a5) as well we need to modify our strategy little bit.

Let

$$
a=x_{n}^{-1} x_{n+1}=x_{n}^{-1} g, b=x_{0}^{-1} x_{1}=x_{1}, U_{1}=\left\{x \in G \mid d\left(x, x_{n}\right)=1, x \neq g\right\} .
$$

Let also $U_{2} \subset B_{2}\left(x_{n}\right) \backslash B_{1}\left(x_{n}\right)$ such that $\left|U_{2}\right|=\left|U_{1}\right|-1=|S|-2$ and for all $x \in U_{1} \backslash\left\{x_{n-1}\right\}$, the set

$$
\left(B_{1}(x) \backslash\{x\}\right) \cap U_{2}
$$

consists of a single element $y(x)$ where $x^{-1} y(x) \notin\left\{a, a^{-1}\right\}$ (See Fig. 3). Finally, we let $U=U_{1} \sqcup U_{2}$ and $V_{0}=\left\{1, x_{1}, \ldots, x_{n-1}\right\} \cup U$. Notice that by minimality assumption on $|g|$, we have $b \neq a^{-1},\left|V_{0}\right|<|G / N|$ and the map $\epsilon$ is still injective on the set $V_{0}$; so the set $V_{0}$ already satisfies conditions (a2), (a3) and (a4). Our goal is to extend $V_{0}$ such that it also satisfies (a1) and (a5). However, notice that $V_{0}$ is not necessarily connected so first we would like to make it connected.

For this purpose, let $S \backslash\{a\}=\left\{g_{1}, \ldots, g_{|S|-1}\right\}$, and for all $1 \leqslant j \leqslant|S|-1$, let $R_{j}$ be a shortest path connecting $x_{n} g_{j}$ to $x_{n-1}$ avoiding $\left\{x_{n}, g\right\}$. Then we let $V=V_{0} \cup \underset{1 \leqslant j \leqslant|S|-1}{\cup} R_{j}$, and observe that $V$ still satisfies conditions (a2), (a3) and (a4) $)^{3}$, moreover, $V$ is connected

\footnotetext{
${ }^{3}$ for the condition (a3), it suffices to notice that $N \cap B_{10 s}(1)=\{1\}$, and recall the condition on the
} 
and $V \cap\left\{x_{n}, g\right\}=\emptyset$.

Let us observe that

$$
\partial V \subseteq\{1\} \sqcup U_{1} \sqcup U_{2} \text { and }\{x \in \partial V \mid x a \in V\} \backslash\left\{x_{n-1}\right\}=\emptyset .
$$

Moreover, if $f V$ is any shift of $V$ disjoint from $V$ then $f V$ cannot contain $x_{n}$. Indeed, if such a shift exists then $x_{n}=f x$ where $x$ must belong to the boundary of $V$, but also $x a$ must be an element of $V$. But since $b \neq a^{-1}$, we cannot have $x=1$. On the other hand, by the definition of $U_{2}$, no element of $U_{2}$ or $U_{1} \backslash\left\{x_{n-1}\right\}$ can be equal to $x$. Finally, (even though $x_{n-1}$ can potentially belong to the set $\{x \in \partial V \mid x a \in V\}$ ) we cannot have $x=x_{n-1}$ because by the structure of $V$, this causes an overlap between $V$ and $f V$.

We let $A_{1}^{\prime}=V, Q_{1}^{\prime}=\epsilon(V)$. Now for all $1 \leqslant k<|G / N|-|V|$ suppose the connected subsets $A_{1}^{\prime}, \ldots, A_{k}^{\prime}$ of $G \backslash\left\{x_{n}, g\right\}$ are already defined such that $\left\{x \in \partial A_{j}^{\prime} \backslash\left\{x_{n-1}\right\} \mid x a \in\right.$ $\left.A_{j}^{\prime}\right\} \backslash\left\{a^{-1}\right\}=\emptyset$ for all $1 \leqslant j \leqslant k$. Then we let $Q_{i}^{\prime}=\epsilon\left(A_{i}\right), 1 \leqslant i \leqslant k$, and build a connected subset $A_{k+1}^{\prime} \supset A_{k}^{\prime}$ such that the conditions

(i) $1 \leqslant\left|A_{k+1}^{\prime} \backslash A_{k}^{\prime}\right| \leqslant 2$,

(ii) $\left\{x \in \partial A_{k+1}^{\prime} \backslash\left\{x_{n-1}\right\} \mid x a \in A_{k+1}^{\prime}\right\} \backslash\left\{a^{-1}\right\}=\emptyset$,

(iii) $A_{k+1}^{\prime} \cap\left\{x_{n}, g\right\}=\emptyset$,

hold.

For this purpose, let

$$
D=\left\{x \in G \mid d\left(x, A_{k}^{\prime}\right)=1\right\}, D^{\prime}=\left\{x \in G / N \mid d\left(x, Q_{k}^{\prime}\right)=1\right\}
$$

and for all $z \in D^{\prime}$, define $C(z)=\left\{x \in Q_{k}^{\prime} \mid d(x, z)=1\right\}$. If there exist $z \in D^{\prime}, y \in$ $D \backslash\left\{x_{n+1}, g\right\}, u \in C(z)$ such that $\epsilon(y)=z$ and $z \neq u \epsilon\left(a^{-1}\right)$, then we define $Q_{k+1}^{\prime}=Q_{k}^{\prime} \cup\{z\}$ and let $A_{k+1}^{\prime}=A_{k}^{\prime} \sqcup\{y\}$.

But if such $z, y$ and $u$ do not exist, then, necessarily, there exist

$$
z_{1}, z_{2} \in(G / N) \backslash Q_{k}^{\prime}, y_{1}, y_{2} \in G \backslash\left(A_{k}^{\prime} \sqcup\left\{x_{n}, g\right\}\right)
$$

such that $\epsilon\left(y_{i}\right)=z_{i}, 1 \leqslant i \leqslant 2, z_{1} \in D^{\prime}, d\left(z_{2}, z_{1}\right)=1$ and $z_{2} \neq z_{1} \epsilon\left(a^{-1}\right) .{ }^{4}$ Then we let $Q_{k+1}^{\prime}=Q_{k}^{\prime} \sqcup\left\{z_{1}, z_{2}\right\}$, and define $A_{k+1}^{\prime}=A_{k}^{\prime} \sqcup\left\{y_{1}, y_{2}\right\}$.

Finally, let $m$ be such that $Q_{m}^{\prime}=G / N$. Then the set $A=A_{m}^{\prime}$ satisfies conditions (a1)-(a5).

Remark 16. Let us emphasize that because of a particular shape of the set $V_{0}$, if it tiles the group $G$ then we have a forced unique partial tiling in a large part of the group. This is because the set $V_{0}$ consists of a "head" $U$ and a "tail" $V_{0} \backslash U$; we have a small "hole" $\left\{x_{n}, g\right\}$ at the head so that in any tiling of $G$ by $V_{0}$ the tail of a shift of $V_{0}$ must enter

generating set $S$ : $S$ has cardinality at least four; there is no relation of length less than four among elements of $S$; and for all distinct $x, y, z \in S$ there exists a path in $G \backslash\{1\}$ of length at most $s$ from $x$ to $y$ not containing $z$.

${ }^{4}$ Indeed, if no such $z, y, u$ exist, then there exists $v \in Q_{k}, z_{1} \in D^{\prime}$ such that $z_{1}=v \epsilon\left(a^{-1}\right)$. Then, again by the non-existence assumption, there is no $w \in Q_{k}$ distinct from $v$ where $d\left(w, z_{1}\right)=1$. Then $z_{1}$ has a neighbor $z_{2}$ such that $z_{2} \neq z_{1} \epsilon\left(a^{-1}\right)$. Lifting $z_{1}, z_{2}$ to $y_{1}, y_{2}$ is straightforward. 
into this hole. We use this observation as a key tool in the proof of Lemma 15. For the proof of the lemma, we need to extend the set $V_{0}$ (the extension is needed to satisfy the condition (a1)) by preserving this distinctive property of it which forces the uniqueness of the tiling. The existence of the tiling follows simply from the conditions (a1) and (a3).

\section{Proof of the Main Theorem}

Let $\mathbb{F}_{2}$ be generated by the set $\{a, b\}$, and $H$ be a virtually non-abelian free quotient of $\mathbb{F}_{2}$ such that there is no relation of length less than four among $a$ and $b$, moreover, for any distinct $x, y, z \in\left\{a, a^{-1}, b, b^{-1}\right\}$ there exists a path $P_{x, y}^{z}$ in $H \backslash\{1\}$ connecting $x$ to $y$ and not containing $z$. Let also $s$ be the maximal length of all these paths $P_{x, y}^{z}$, for all distinct $x, y, z \in\left\{a, a^{-1}, b, b^{-1}\right\}$.

Let us observe that $H$ possesses a connected partial tile with Heesch number zero. Indeed, the set $B_{s+1}(1) \backslash\{1\}$ is connected and no non-overlapping shift of it can cover the identity element.

For the proof, we will construct the group $\Gamma$ as a hyperbolic limit of the group $H$ with a fixed generating set $S=\left\{a, a^{-1}, b, b^{-1}\right\}$. We will build the hyperbolic limit of $H=H_{0}$ inductively as follows. Suppose the groups $H_{0}, \ldots, H_{n}$ have been constructed such that the following conditions hold:

(i) $H_{i}$ is a quotient of $H_{i-1}$, for all $1 \leqslant i \leqslant n$;

(ii) $H_{i}$ is virtually non-abelian free, for all $0 \leqslant i \leqslant n$;

(iii) $H_{i}$ possesses a partial tile $K_{i}$ of finite Heesch number at least $i$, for all $0 \leqslant i \leqslant n$;

(iv) For all $1 \leqslant i \leqslant n$, the quotient epimorphism $\pi_{i}: H_{i-1} \rightarrow H_{i}$ is injective on the ball of radius $(i+1)(r(i)+1)$ around the identity element w.r.t. the generating set $S$ where $r(i)=\max \left\{|g|_{j} \mid 0 \leqslant j \leqslant i-1, g \in K_{j}\right\}, \forall i \geqslant 1$ and $|\cdot|_{i}$ denotes the left-invariant Cayley metric in $H_{i}$ with respect to the generating set $S$ (we let $r(0)=10 s$ ).

Let us notice that conditions (i)-(iv) hold for $n=0$ (in fact, conditions (i) and (iv) are void, the condition (ii) is true by our choice of $H_{0}$, and we already established that the condition (iii) is satisfied).

Now, by Lemma 10 there exists a normal subgroup $N \unlhd H_{n}$ of a finite index such that $N$ is a free group of rank $k \geqslant 4$ generated by elements $a_{1}, \ldots, a_{k}$ and $\min \left\{\left|a_{1}\right|_{n}, \ldots,\left|a_{k}\right|_{n}\right\} \leqslant$ $1.1 \min \left\{|x|_{n} \mid x \in N \backslash\{1\}\right\}$ (i.e. conditions (c1) and (c2) of Lemma 10 hold). Without loss of generality we may assume that

$$
\left|a_{1}\right|_{n}=\min \left\{\left|a_{1}\right|_{n}, \ldots,\left|a_{k}\right|_{n}\right\} .
$$

Then, by Lemma 15, there exists a connected set $A \subset H_{n}$ satisfying conditions (a1)-(a5). (In applying this lemma we take $g=a_{1}$; then, in particular, $d\left(a_{1}, u\right)=1$ for some $u \in A$ ).

Let also

$$
R_{1}=\max \left\{|g|_{n} \mid g \in K_{i}, 1 \leqslant i \leqslant n\right\}, R_{2}=\max \left\{\left|a_{1}^{j}\right|_{n} \mid 1 \leqslant j \leqslant 10(n+1)\right\} .
$$

By Proposition 11 there exists a generating set $\left\{h_{1}, \ldots, h_{k}\right\}$ of $N$, and an odd number $p_{n} \geqslant 1$ such that the quotient

$$
H_{n+1}:=H_{n} /\left\langle h_{i}^{p_{n}}=1,1 \leqslant i \leqslant k\right\rangle
$$


is a virtually non-abelian free group, and the quotient epimorphism

$$
H_{n} \rightarrow H_{n} /\left\langle h_{i}^{p_{n}}=1,1 \leqslant i \leqslant k\right\rangle
$$

is injective on the ball of radius $R:=\max \left\{R_{1}^{n}, R_{2}\right\}$ around the identity element. Then notice that, by Lemma 9, all the partial tiles $K_{1}, \ldots, K_{n}$ inject into $H_{n+1}$, moreover, the images of $K_{i}$ have a finite Heesch number at least $i+1$ in $H_{n+1}$. Then we take $K_{n+1}=A \cup a_{1} A$. Notice that $K_{n+1}$ has a Heesch number at least $n+1$.

It remains to notice that by condition (a5) the Heesch number of $K_{n+1}$ in $H_{n+1}$ is at most $\frac{p_{n}}{2}$ thus it is finite. Thus we can continue the inductive process (for the constructed group $H_{n+1}$ we verified all the conditions (i)-(iv) for $i=n+1$ ). Then the hyperbolic limit group $H_{\infty}$ will be a group with desired properties, i.e. the connected sets $K_{1}, K_{2}, \ldots$ will be all partial tiles with finite Heesch numbers $k_{1}, k_{2}, \ldots$ such that $k_{n} \geqslant n$ for all $n \geqslant 1$.

\section{Acknowledgements}

I am thankful to I.Pak for encouraging me to write this paper, and to C.Mann for giving me a permission to use the figures in his website (Figure 1,2). I am also thankful to the anonymous referees for several very useful suggestions.

\section{References}

[1] P. de la Harpe and E. Ghys. Sur les groupes hyperboliques d'aprés Mikhael Gromov. Birkhäuser, 1990.

[2] T. Delzant. Sous-groupes distingues et quotients des groupes hyperboliques. Duke Math. J., 83(3):661-682, 1996.

[3] A. Fontaine. An infinite number of plane figures with heesch number two, J. Combin. Theory Ser. A, 57:151-156, 1991.

[4] M. Gromov. Essays in Group Theory, Math. Sci. Research Institue Publications, 8. edited by S.M.Gersten. 1987.

[5] H. Heesch. Regulares Parkettierungsproblem, Westdeutscher Verlag, Cologne, 1968.

[6] C. Mann. Heesch's Problem and Other Tiling Problems, Ph.D. dissertation, University of Arkansas at Fayetteville, 2001.

[7] C. Mann. Heesch's tiling problem American Mathematical Monthly, 111(6):509-517, 2004.

[8] G. Margulis and S. Mozes. Aperiodic tilings of the hyperbolic plane by convex polygons. Israel J. Math., 107: 319-325, 1998.

[9] J. Socolar and J. M. Taylor. An aperiodic hexagonal tile, J. Combin. Theory, Ser. A, 118(8):2207-2231, 2011.

[10] J. Socolar and J. M. Taylor. Forcing nonperiodicity with a single tile, The Mathematical Intelligencer, 34(1):18-28, 2012. 
[11] A. S. Tarasov. On the Heesch number for the hyperbolic plane. Mathematical Notes, 88(1):97-104, 2010.

[12] B. Zimmermann. Finite groups of outer automorphisms of free groups. Glasgow Math. J., 38(3):275-282, 1996. 